\title{
USO DE ANÁLISE DE ENVOLTÓRIA DE DADOS EM AGRICULTURA: O CASO DE HOLAMBRA
}

\author{
Eliane Gonçalves Gomes ${ }^{1}$ \\ João Alfredo de Carvalho Mangabeira ${ }^{2}$
}

\begin{abstract}
Resumo: O uso de Análise de Envoltória de Dados (DEA) para medir eficiência relativa de agricultores pode servir como apoio à decisão na produção agrícola, ao indicar as fontes de ineficiência e as unidades que podem servir de referência às práticas adotadas. Este artigo usa DEA para medir a eficiência de 71 agricultores do município de Holambra, estado de São Paulo. Os dados utilizados referem-se ao ano de 2002.
\end{abstract}

Palavras-chave: Análise de Envoltória de Dados; Eficiência Técnica; Agricultura.

Abstract: Data Envelopment Analysis (DEA) when applied to evaluate farmers' relative efficiency can support decision-making, as it indicates inefficiencies sources and the benchmarks. This paper employs DEA to measure the efficiency of 71 farmers from Holambra, São Paulo State. The data used are from year 2002.

Key-words: Data Envelopment Analysis; Technical efficiency; Agriculture.

\section{INTRODUÇÃO}

O cálculo da eficiência de unidades produtivas tem importância tanto para fins estratégicos, quanto para o planejamento e para a tomada de decisão.

A eficiência de uma unidade produtiva é medida através da comparação entre os valores observados e os valores ótimos de seus produtos (saídas) e recursos (insumos). Esta comparação pode ser feita, em linhas gerais, pela razão entre a produção observada e a produção potencial máxima alcançável, dados os recursos disponíveis, ou pela razão entre a quantidade mínima necessária de recursos e a quantidade efetivamente empregada, dada a quantidade de produtos gerados. Combinações dessas razões podem igualmente prover informações importantes.
A mensuração dessas medidas de desempenho é feita via envelopamento dos dados, limitados por regiões (fronteiras) de máxima produção ou de mínimo custo. Esta modelagem envolve uma boa variedade de técnicas econométricas (fronteiras de produção) e de programação matemática. Neste último caso, os modelos existentes são os que recebem a denominação de modelos de Análise de Envoltória de Dados (Data Envelopment Analysis - DEA ).

$O$ uso de DEA para medir a eficiência relativa de unidades produtivas tem-se mostrado bastante atrativo em diversos setores de aplicação. O emprego de modelos DEA em agricultura pode apoiar as decisões dos agricultores, ao indicar as fontes de ineficiência e as unidades que podem servir de referência às práticas adotadas (benchmarks). Gomes et al. (2003a) apresentam uma revisão sobre o estado da arte do uso de

1,2 Embrapa Monitoramento por Satélite, E-mail: ${ }^{1}$ eliane@cnpm.embrapa.br; ${ }^{2}$ manga@cnpm.embrapa.br 
DEA em agricultura, bem como sua integração com Sistemas de Informação Geográfica.

Este artigo faz uso de DEA para medir a eficiência de uma amostra de 71 agricultores do município de Holambra, estado de São Paulo. Os dados utilizados, originalmente usados para tipificação dos produtores rurais (MANGABEIRA, 2002), referem-se ao ano de 2002 e foram obtidos por levantamento de campo, via questionários socioeconômicos.

\section{ANÁLISE DE ENVOLTÓRIA DE DADOS}

No estudo dos temas produtividade e eficiência em agricultura pode surgir a seguinte questão: existe erro em usar toneladas de grão por hectare como medida de eficiência de um produtor rural? Medidas desse tipo apresentam a deficiência de não considerarem outros recursos na medida de eficiência, como mão-de-obra, maquinarias, combustível, fertilizantes etc. (GOMES et al., 2003a). O uso dessa medida na formulação de políticas pode resultar no uso excessivo dos recursos não incluídos na medida de eficiência. A abordagem por Análise de Envoltória de Dados (Data Envelopment Analysis DEA), que utiliza programação linear para estimar a fronteira eficiente (linear por partes), é capaz de incorporar diversos inputs (entradas, recursos, insumos ou fatores de produção) e outputs (saídas ou produtos) para o cálculo da eficiência de unidades tomadoras de decisão, designadas por DMUs (Decision Making Units).

Os problemas de programação linear (PPLs) de DEA otimizam cada observação individual com o objetivo de calcular uma fronteira de eficiência, determinada pelas unidades que são Pareto-eficientes.

Há dois modelos DEA clássicos. O modelo CCR (também conhecido por CRS ou constant returns to scale), que trabalha com retornos constantes de escala (CHARNES et al., 1978) e assume proporcionalidade entre inputs e outputs. O modelo BCC (ou VRS), devido a Banker et al. (1984), considera retornos variáveis de escala, isto é, substitui o axioma da proporcionalidade pelo axioma da convexidade.

Tradicionalmente são possíveis duas orientações para esses modelos: orientação a inputs, quando deseja-se minimizar os recursos disponíveis, sem alteração do nível de produção; orientação a outputs, quando o objetivo é aumentar os produtos, sem mexer nos recursos utilizados.

Existem duas formulações equivalentes para DEA. De forma simplificada, pode-se dizer que uma das formulações (modelo do Envelope) define uma região viável de produção e trabalha com uma projeção de cada DMU na fronteira dessa região. A outra formulação (modelo dos Multiplicadores) trabalha com a razão de somas ponderadas de produtos e recursos, com a ponderação escolhida de forma mais favorável a cada DMU, respeitando-se determinadas condições.

Neste artigo, o modelo DEA a ser usado é o BCC. Em (1) e em (2) apresentam-se, respectivamente o modelo DEA BCC dos Multiplicadores e do Envelope, com orientação a outputs. Considera-se que cada DMU $k, k=1 . . . n$, é uma unidade de produção que utiliza $m$ inputs $x_{i k}, i=1 \ldots m$, para produzir $s$ outputs $y_{j k}, j=1 \ldots$ s. Em ambas as formulações $h_{o}$ é a eficiência da DMU $o$ em análise; $x_{i o}$ e $y_{j o}$ são os inputs e outputs da DMU o. Em (1), $v_{i}$ e $u_{j}$ são os pesos calculados pelo modelo para inputs e outputs, respectivamente; $v_{*}$ é a variável dual associada à condição $\sum_{k} \lambda_{k}=1$ da formulação (2) e é interpretado como fator de escala: quando positivo, indica retornos decrescentes de escala; quando negativo, indica retornos crescentes de escala; caso seja nulo, a situação é de retornos constantes de escala. Em (2), $\lambda_{k}$ representa a contribuição da DMU $k$ na formação do alvo da DMU $o$. 
Min $h_{o}=\sum_{i=1}^{m} v_{i} x_{i o}-v_{*}$

sujeito a

$\sum_{j=1}^{s} u_{j} y_{j o}=1$

$-\sum_{i=1}^{m} v_{i} x_{i k}+\sum_{j=1}^{s} u_{j} y_{j k}+v_{*} \leq 0, \forall k$

$u_{j} \geq 0, v_{i} \geq 0, \forall i, j$

$\nu_{*} \in \mathfrak{R}$

$\operatorname{Max} h_{o}$

sujeito a

$x_{i o}-\sum_{k=1}^{n} x_{i k} \lambda_{k} \geq 0, \forall i$

$-h_{o} y_{j o}+\sum_{k=1}^{n} y_{j k} \lambda_{k} \geq 0, \forall j$

$\sum_{k=1}^{n} \lambda_{k}=1$

$\lambda_{k} \geq 0, \forall k$

3. AGRICULTURA EM HOLAMBRA

O município de Holambra situa-se a $145 \mathrm{~km}$ da cidade de São Paulo, na região centro leste do Estado, a $22^{\circ} 37^{\prime} 55^{\prime \prime}$ de latitude sul e $47^{\circ} 03{ }^{\prime} 36^{\prime \prime}$ de longitude oeste (Figura 1).

Tem aproximadamente $65 \mathrm{~km}^{2}$, com uma altitude média de $600 \mathrm{~m}$, e é banhado pelos rios Jaguari, Camanducaia e Pirapitingui.

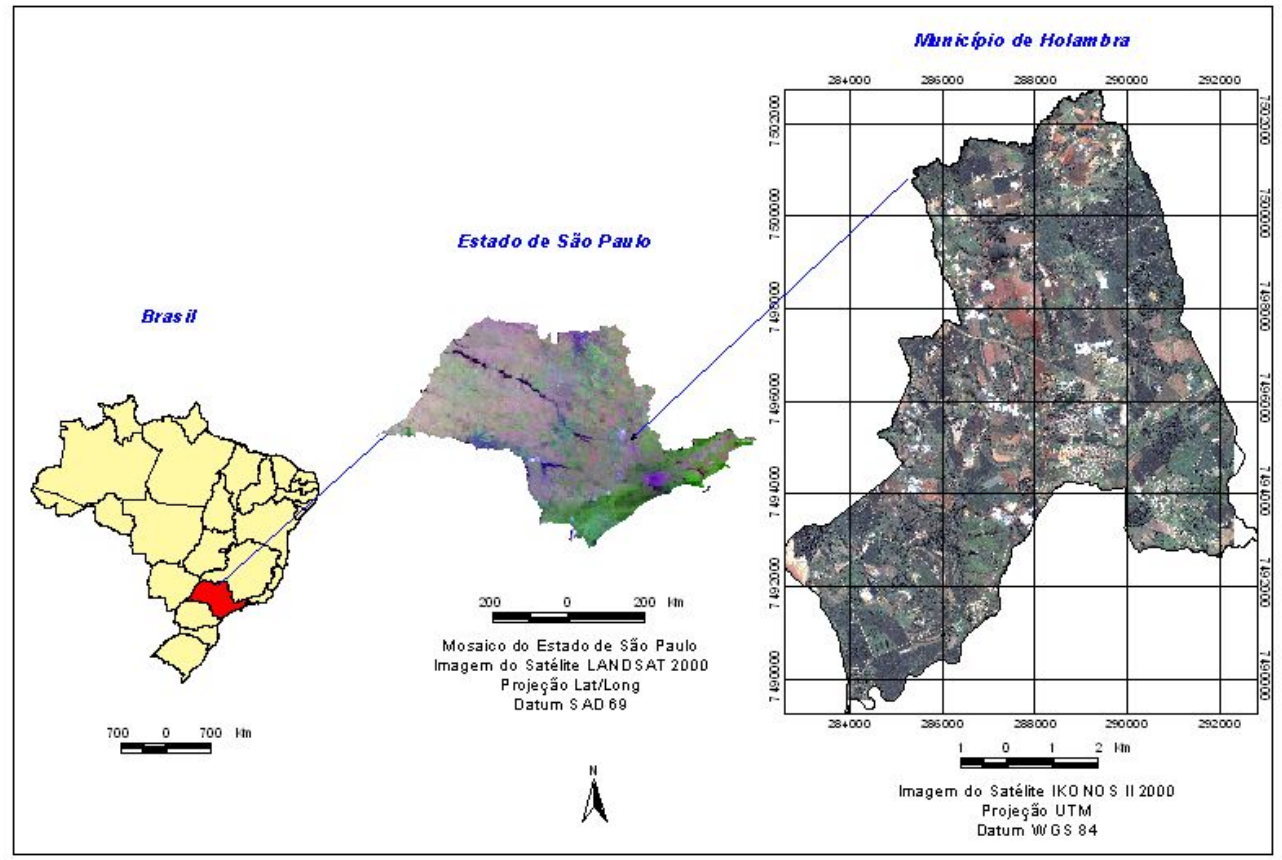

Figura 1. Localização do Município de Holambra. 
Holambra é limitado pelos municípios de Artur Nogueira, Cosmópolis, Santo Antônio da Posse e Jaguariúna. As duas principais rodovias que passam pelo município são a SP-340 (Adhemar de Barros) e a SP-107. Sua população é de 7.211 habitantes, sendo 3.689 homens e 3.522 mulheres, segundo o Censo Demográfico do IBGE de 2000 (http://www.ibge.gov.br/cidadesat/default .php).

A economia do município é praticamente baseada na atividade agropecuária, com predomínio das atividades hortícolas, citrícolas, plantas decorativas, flores, suinocultura, avicultura e laticínios. Segundo o Censo Agropecuário do IBGE de 1996 (http://www.sidra.ibge.gov.br), naquele ano 0 município detinha aproximadamente 1.466,3 ha de culturas temporárias, $1.769,5$ ha de culturas permanentes, 432,4 ha de pastagens, 89,6 ha de matas e florestas naturais e 13,81 ha de reflorestamentos. Possuía ainda um efetivo de aproximadamente 1.800 bovinos, 28.000 suínos e aproximadamente 792.000 aves.

O município apresenta dimensão territorial relativamente pequena em relação aos demais municípios brasileiros e quase toda sua produção agrícola advém de um grupo aproximado de 287 produtores, com 20 ha em média de área (HOLAMBRA, 1998).

Mangabeira (2002) mapeou o uso das terras do município de Holambra com base em imagens do satélite Ikonos II (do ano de 2001) e levantamentos de campo (em 2002) (Figura 2). Com o uso de questionários de campo, levantou informações de 266 variáveis socioeconômicas (que geraram 204 indicadores), para uma amostra de 74 produtores rurais (Figura 3).

Com esses indicadores foi possível tipificar essa amostra de produtores. Para tal foi usada a Análise de Correspondência Múltipla (BISHOP et al., 1975), seguida da análise de cluster pelo método de Ward (EVERITT, 1974), para classificar os tipos de produtores agrícolas. Os resultados (MANGABEIRA, 2002; AZEVEDO et al., 2001) mostraram a existência de 6 tipos de produtores no município: produtores agrianuais, floricultores, agrocitricultores, florescitricultores, agropecuaristas e agrofloricultores. 


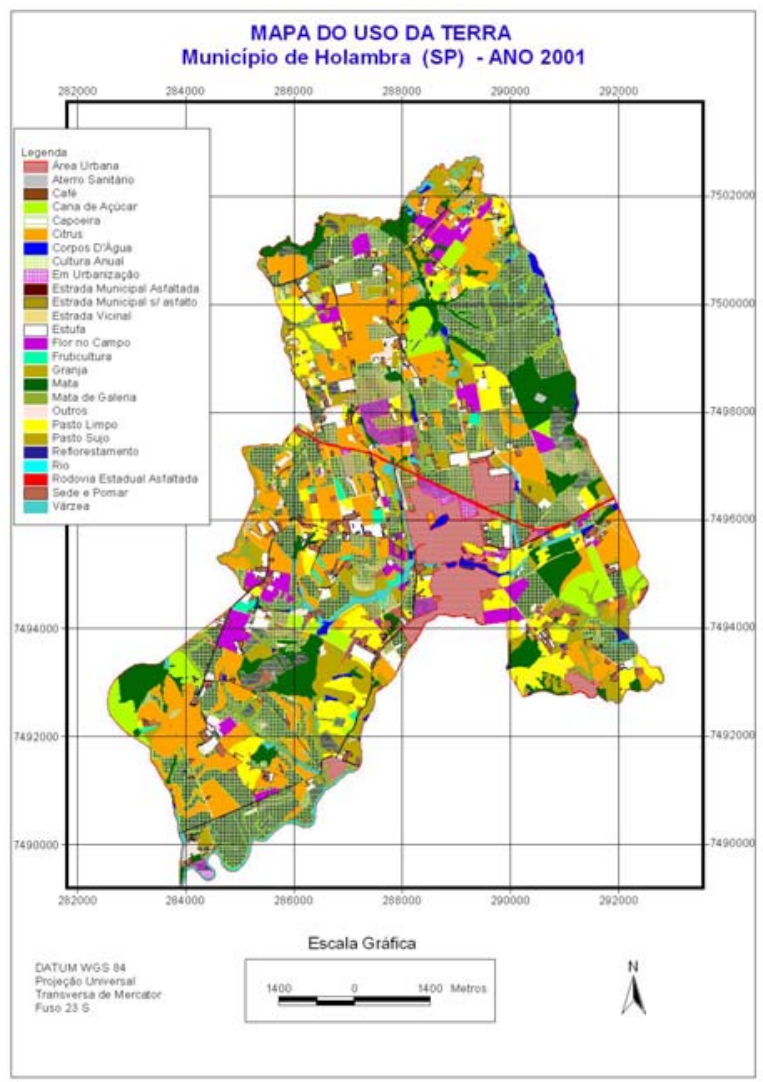

Figura 2. Mapeamento do uso das terras de Holambra.

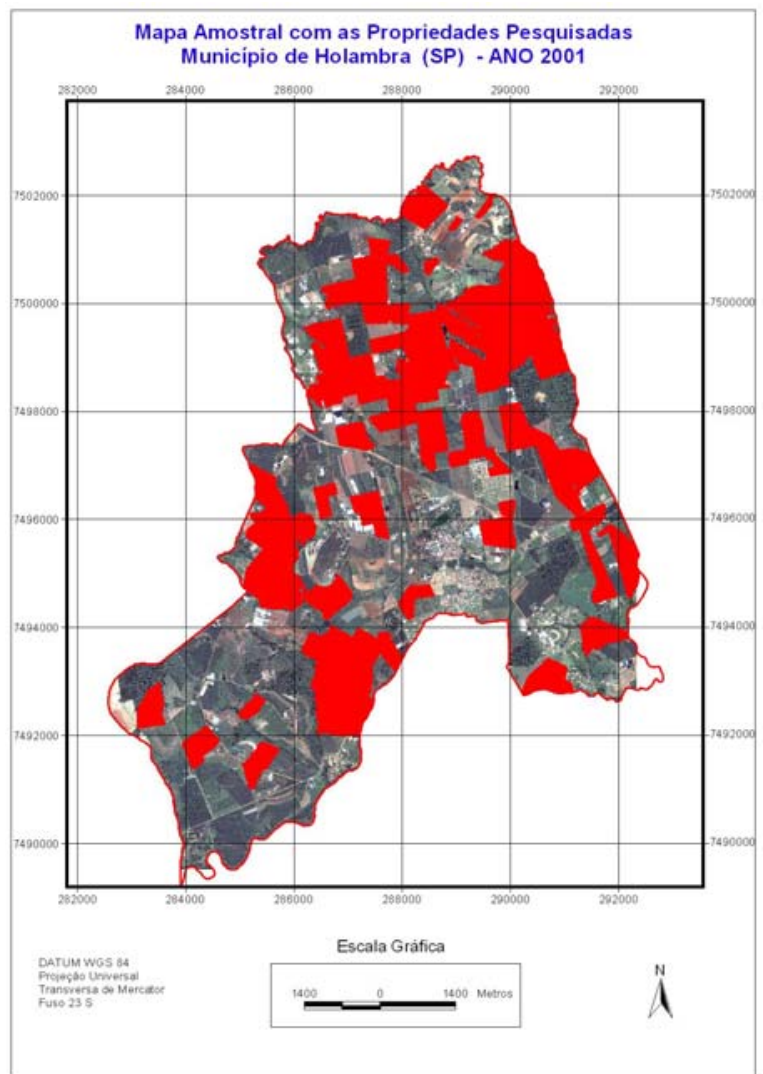

Figura 3. Distribuição espacial das propriedades amostradas para o levantamento com questionários de campo.

4. MODELAGEM E RESULTADOS

\subsection{Estruturação do problema}

ENGEVISTA, v. 6, n. 1, p. 19-27, abr. 2004
Para estruturar um modelo DEA é necessário definir as unidades a serem investigadas, as variáveis de avaliação 
(inputs e outputs) e o modelo DEA adotado (CCR, BCC, entre outros; orientação a inputs, a outputs etc.).

Na construção do modelo DEA deve-se atentar quais variáveis serão consideradas inputs e quais serão consideradas outputs. Muitas vezes uma variável representa algo que é produzido, mas cuja quantidade deve ser minimizada (ex. acidentes, poluição). Nesses casos, essa variável será, na realidade, tratada como um input. De forma geral, as variáveis que se desejam maximizar são consideradas outputs, e inputs caso contrário.

Um modelo com grande número de variáveis pode acarretar outro problema, qual seja, uma avaliação extremamente benevolente, com várias DMUs $100 \%$ eficientes. Existe uma recomendação empírica que o número de DMUs seja pelo menos o dobro ou o triplo do número de variáveis. Estudos mais recentes (González-Araya, 2003) indicam que essa relação deve ser ainda maior (4 a 5 vezes), em especial quando, além do índice de eficiência, deseja-se analisar os benchmarks das unidades em avaliação.

Para a avaliação dos agricultores de Holambra foram selecionados 4 dos 206 indicadores derivados por Mangabeira (2002). Essas variáveis indicam as relações clássicas entre produção, capital e trabalho. Como inputs foram selecionados Área Cultivada (representada pela área ocupada com cultivos mais a área de pasto), em hectares, Emprego (expresso por homemhora-ano) e Máquinas (calculado como número total de horas de uso de máquinas no ano); Renda Líquida anual da propriedade, em reais, é o output. Destaca-se que em casos em que as relações causais não são claras ou quando há diversas variáveis que podem ser consideradas no modelo, métodos adicionais para seleção de variáveis devem ser empregados, como por exemplo, aqueles indicados por EstellitaLins e Moreira (1999), Soares de Mello et al. (2002b, 2004a) e Galagedera e Silvapulle (2003).
As unidades de avaliação (DMUs) são os agricultores amostrados por Mangabeira (2002). Dos 74 produtores, 3 (produtores de leite) apresentaram valor negativo para a variável Renda Líquida e foram descartados da análise.

O modelo selecionado foi o modelo DEA BCC orientado a outputs, já que há diferenças de escala significativas entre as DMUs e o objetivo é maximizar a renda do produtor, ou seja, obter produção máxima dadas as condições de trabalho.

\subsection{Resultados}

Os resultados do modelo DEA foram obtidos com o uso do software SIAD (ANGULO-MEZA et al., 2003). Dos 71 produtores avaliados, 6 foram $100 \%$ eficientes, dos quais 4 são floricultores. A eficiência média da amostra foi de 23,8\%; para os produtores de flores, a eficiência média foi de $24,8 \%$ e de $22,4 \%$ para os demais.

Analisando-se os resultados do modelo dos multiplicadores (pesos e fatores de escala), verifica-se uma grande quantidade de pesos zero, em especial para a variável Área Cultivada (88,7\% das DMUs). Isto significa que no cálculo da eficiência esta variável foi desconsiderada para que a DMU obtivesse a eficiência máxima possível, quando comparada com as demais. Isso mostra que poucos agricultores fazem bom uso da área, o que pode igualmente explicar o baixo valor de eficiência média. O problema dos pesos zero pode ser contornado com o emprego de modelos adicionais, como, por exemplo, avaliação cruzada (SEXTON et al., 1986; DOYLE E GREEN, 1995), restrições aos pesos (ALLEN et al., 1997) e suavização da fronteira DEA (SOARES DE MELLO et al., 2002a, 2004b; SOARES DE MELLO, 2002).

Ainda em relação ao modelo dos multiplicadores, 3 DMUs apresentarem retornos constantes de escala, 10 decrescentes e o restante retornos crescentes de escala. Isto mostra que para a maioria das DMUs, acréscimos na utilização de insumos (inputs) acarretam 
aumentos mais que proporcionais na geração de produtos.

Os resultados do modelo do envelope (benchmarks e alvos) mostram que o produtor eficiente que aparece $o$ maior número de vezes como referência para as outras unidades é um pequeno produtor de flores, com valores de área cultivada e emprego menores que a média destes produtores (367\% e 163\%, respectivamente) e valores de uso de máquinas e renda líquida superiores à média (24\% e $87 \%)$. Essa DMU pode servir como exemplo a ser seguido pelas unidades ineficientes, em especial, no que se refere às práticas de gestão das propriedades.

Verifica-se ainda que diversas DMUs projetam-se em faces constituídas por DMUs eficientes que são floricultores e não floricultores. Isto torna-se importante na promoção da eficiência das DMUs ineficientes, já que estas devem observar não somente as práticas de seus "concorrentes diretos", mas também aquelas dos demais produtores da região.

Em relação aos alvos, destaca-se a contribuição principal dos modelos DEA para a agricultura, qual seja, informar ao agricultor quais são as fontes de ineficiência e o que deve ser feito para a busca da eficiência. Por exemplo, para uma DMU que obteve $69,6 \%$ de eficiência, a recomendação para a busca da eficiência é o aumento em 43,7\% da renda líquida, dados os inputs de que dispõe. Desta forma, esse agricultor deve verificar em etapa de seu processo produtivo (outras variáveis não consideradas) ocorrem os gargalos que prejudicam seus resultados.

Para uma segunda DMU, com eficiência de 74,2\%, as diretrizes são diferentes. Para alcançar a eficiência, esta deve reduzir a área cultivada, o nível de emprego e o uso de máquinas em, respectivamente, $99,5 \%, 85,0 \%$ e $83,0 \%$, e aumentar a renda líquida em 34,8\%. Neste ponto, cabe destacar que o modelo DEA utilizado permite que haja redução em inputs que podem estar fora do controle do agricultor, como por exemplo, área. Neste caso, o uso de modelos não radiais, que não permitissem alteração nesta variável, seriam mais indicados. Entretanto, neste artigo, este caminho não foi o adotado.

Um outro ponto a ressaltar é a possibilidade de o agricultor não ser capaz de alcançar de forma imediata o alva que lhe é atribuído. Neste caso, uma busca seqüencial por alvos intermediários, conforme apresentado por Gomes et al. (2003b) e Gomes (2003), pode ser uma solução atrativa ao produtor rural para a busca de alvos realizáveis, ou seja, mais próximos à sua realidade.

Outro destaque deve ser dado à projeção de DMUs ineficientes em regiões não Pareto-eficientes, o que é verificado pela presença de folgas diferentes de zero. Para a projeção em somente faces Pareto-eficientes, modelos DEA avançados devem ser empregados como aqueles usam programação multiobjetivo para seleção de alvos (ANGULO-MEZA, 2002), que projetam as unidades ineficientes em faces de maior dimensão (GONZÁLEZ-ARAYA, 2003) e que eliminam as regiões não Pareto-eficientes por meio de suavização da fronteira DEA (SOARES DE MELLO, 2002).

\section{CONCLUSÕES}

O emprego do modelo DEA à avaliação dos agricultores de Holambra mostrou-se bastante interessante, em especial no que se refere à determinação de diretrizes aos agricultores ineficientes para o alcance da eficiência (redução dos recursos e/ou aumento da produção). Além disso, a indicação de unidades referência ou benchmarks é importante na mudança das práticas de gestão das propriedades.

Modelos DEA avançados podem melhorar os resultados obtidos, seja na redução dos pesos zero, seja na possibilidade de seleção de alvos a alcançar. Esses desenvolvimentos serão contemplados em estudos futuros.

\section{REFERÊNCIAS BIBLIOGRÁFICAS}


ALLEN, R., ATHANASSOPOULOS, A., DYSON, R.G., THANASSOULIS, E. Weights restrictions and value judgements in data envelopment analysis: evolution, development and future directions. Annals of Operations Research, n. 73, p. 13-34, 1997.

ANGULO-MEZA, L., BIONDI NETO, L., SOARES DE MELLO, J.C.B., GOMES, E.G., COELHO, P.H.G. SIAD - Sistema Integrado de Apoio à Decisão: Uma Implementação Computacional de Modelos de Análise Envoltória de Dados. Relatórios de Pesquisa em Engenharia de Produção, v. 3, n. 20. 2003. Disponível em: http://www.producao.uff.br/rpep/rel pesq303/relpesq_303_20.doc.

Acesso em: 5 jan. 2004.

ANGULO-MEZA, L. Um enfoque multiobjetivo para os modelos de determinação de alvos em DEA. Rio de Janeiro, 2002. Tese (Doutorado em Engenharia de Produção) - COPPE, Universidade Federal do Rio de Janeiro.

AZEVEDO, E.C. de, MANGABEIRA, J.A. de C., MIRANDA, J.R. Análise da Sustentabilidade das Atividades Agrícolas: Uma Contribuição dos Sistemas de Informações Geográficas na Gestão Ambiental e no Desenvolvimento Sustentado. In: CONGRESSO BRASILEIRO DE ADMINISTRAÇÃO RURAL, 4., 2001, Goiânia. Anais... Lavras: ABAR, 2001. 8p.

BANKER, R.D., CHARNES, A., COOPER, W.W. Some models for estimating technical scale inefficiencies in Data Envelopment Analysis. Management Science, v. 30, n. 9, p. 1078-1092, 1984.

BISHOP, Y., FIENBERG, S., HOLLAND, P. Discrete multivariate analysis: theory and practice. Cambridge, Estados Unidos: MIT, 1975. 575 p.

CHARNES, A., COOPER, W.W., RHODES, E. Measuring the efficiency of decision-making units. European Journal of Operational Research, v. 2, p. 429-444, 1978.

DOYLE, J.R., GREEN, R.H. Crossevaluation in DEA: Improving discrimination among DMU's. INFOR, v. 33, n. 3, p. 205-222, 1995.

ESTELLITA-LINS, M.P., MOREIRA, M.C.B. Método I-O Stepwise para Seleção de Variáveis em Modelos de Análise Envoltória de Dados. Pesquisa Operacional, v. 19, n. 1, p. 39-50, 1999.

EVERITT, B. Cluster analysis. London: Heinemann Educational, 1974. 122 p.

GALAGEDERA, D., SILVAPULLE, P. Experimental evidence on robustness of data envelopment analysis. Journal of the Operational Research Society, v. 54, p. 654-660, 2003.

GOMES, E.G. Modelos de Análise de Envoltória de Dados com Ganhos de Soma Zero. Rio de Janeiro, 2003. Tese (Doutorado em Engenharia de Produção) COPPE, Universidade Federal do Rio de Janeiro.

GOMES, E.G., SOARES DE MELLO, J.C.C.B., BIONDI NETO, L. Avaliação de eficiência por Análise de Envoltória de Dados: conceitos, aplicações à agricultura e integração com Sistemas de Informação Geográfica. Documentos, n. 28. Campinas: Embrapa Monitoramento por Satélite, 2003a.

GOMES, E.G., SOARES DE MELLO, J.C.C.B., ESTELLITA-LINS, M.P. Busca seqüencial de alvos intermediários em modelos DEA com soma de outputs constante. Investigação Operacional, v. 23, n. 2, 2003b (no prelo).

GONZÁlEZ-ARAYA, M.C. Projeções não radiais em regiões fortemente eficientes da fronteira DEA Algoritmos e Aplicações. Rio de Janeiro, 2003. Tese (Doutorado em Engenharia de Produção) - 
COPPE, Universidade Federal do Rio de Janeiro.

HOLAMBRA em números. Holambra Hoje, agosto, 1998. (3 ${ }^{\text {a }}$ capa).

MANGABEIRA, J.A. de C. Tipificação de Produtores Rurais Apoiada em Imagens de Alta Resolução Espacial, Geoprocessamento e Estatística Multivariada: Uma Proposta Metodológica. Campinas, 2002. Dissertação (Mestrado em Planejamento e Desenvolvimento Rural Sustentável) - Faculdade de Engenharia Agrícola, UNICAMP.

SEXTON, T.R., SILKMAN, R.H., LOGAN A.J. Data Envelopment Analysis: Critique and extensions. In: .H. Silkman (Ed.) Measuring Efficiency: An Assessment of Data Envelopment Analysis. Jossey-Bass Editor, San Francisco, 1986.

SOARES DE MELLO, J.C.C.B. Suavização da fronteira DEA com o uso de métodos variacionais. Rio de Janeiro, 2002. Tese (Doutorado em Engenharia de Produção) - COPPE, Universidade Federal do Rio de Janeiro.

SOARES DE MELLO, J.C.C.B., ESTELLITA-LINS, M.P., GOMES, E.G. Construction of a smoothed DEA frontier. Pesquisa Operacional, v. 22, n. 2, p. 183201, 2002a. Disponível em: http://www.scielo.br/pdf/pope/v22n 2/a06v22n2.pdf. Acesso em: 5 mar. 2004.

SOARES DE MELLO, J.C.B., GOMES, E.G., SOARES DE MELLO, M.H.C, ESTELLITA-LINS, M.P. Método Multicritério para Seleção de Variáveis em modelos DEA. Revista Pesquisa Naval, v. 15, p. 55-66, $2002 b$.

SOARES DE MELLO, J.C.B, GOMES, E.G., ANGULO-MEZA, L., ESTELLITA-LINS, M.P. Selección de variables para el incremento del poder de discriminación de los modelos DEA. Revista Epio Escuela de Perfeccionamiento En
Investigación Operativa, n. 24, 2004a (no prelo).

SOARES DE MELLO, J.C.C.B., GOMES, E.G., BIONDI NETO, L., ESTELLITA-LINS, M.P. Suavização da fronteira DEA: o caso BCC tridimensional. Investigação Operacional, v. 24, n. 1, 2004b (no prelo). 